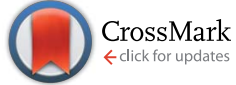

Cite this: J. Anal. At. Spectrom., 2016 31, 1198

Received 11th February 2016 Accepted 5th May 2016

DOI: $10.1039 / c 6 j a 00042 \mathrm{~h}$

www.rsc.org/jaas

\section{A calibration strategy for LA-ICP-MS using isotope dilution for solid reference materials}

\author{
J. P. Thieleke and C. Vogt
}

An isotope dilution method for the quantitative analysis of trace elements in biological samples and for the characterization of reference materials for the analysis of biological samples by laser ablation-inductively coupled plasma-mass spectrometry (LA-ICP-ID-MS) has been tested. Polymeric thin layers with 5-10 $\mu \mathrm{m}$ thickness containing the ID-spike and the correction standard were produced by knife application onto polyester substrates. For the ID experiments ${ }^{204} \mathrm{~Pb}$ enriched lead was used, and bismuth served as the internal standard. The layers showed good homogeneity of the layer thickness and the distribution of added elements. For the testing of the ID method, a synthetic sample with lead and a lead containing polymer certified reference material (BAM H010) have been used. Mass fractions for lead calculated after single and double isotope dilution experiments in the test samples showed a deviation of less than $1 \%$ from the certified values.

\section{Introduction}

The spatially resolved analysis of element distributions in biological samples is of significant scientific interest. The localization and quantification of trace elements in biological tissues can provide information on routes of exposure, accumulation and distribution of essential and toxic trace elements. Too low concentrations of certain elements may have negative effects on cell growth, while too high concentrations are toxic. Furthermore, some trace elements are indicators of diseases or deficiencies. ${ }^{\mathbf{1 - 4}}$ Nowadays several analytical methods are applied for the spatially resolved investigation of trace element distribution, among them are $\mu \mathrm{XRF},{ }^{5-10}$ SIMS $^{10-14}$ and LA-ICP-MS. ${ }^{\mathbf{1 0 1 5 , 1 6}}$ In comparison with the other methods LA-ICP-MS offers several advantages, such as working under non-vacuum conditions, low limits of detection and in general relatively fast quantification. Nevertheless an accurate quantification of trace element concentrations in biomaterials by LA-ICP-MS is challenging; since the sample matrix affects the process of generation of the analytical signal, the sample composition may vary significantly within a few micrometers and the appointment of an element for internal standardization is often hampered by the non-uniform distribution of all elements within the sample. Furthermore, certified reference materials are hardly available for the analysis of specific biological samples. Therefore the development of an accurate in-house characterization process for self-prepared standards is useful.

Thus, for the calibration of trace metal concentrations in biomaterials by LA-ICP-MS, different calibration strategies are

University of Hannover, Institute of Inorganic Chemistry, Callinstr. 9, 30167 Hannover, Germany. E-mail: thieleke@acc.uni-hannover.de published, for example, internal standardization, ${ }^{17}$ external calibration, ${ }^{\mathbf{1 8 , 1 9}}$ matrix matched standards, ${ }^{\mathbf{2 0 , 2 1}}$ solution based isotope dilution ${ }^{22-25}$ (ID) and film coating. ${ }^{25}$ The results differ in the accuracy of quantification depending on the calibration method used. In most cases, accuracies between 10 and $20 \%$ were obtained which are typical for most quantification procedures in solid state spectroscopy. Nevertheless better statistical data are sometimes of the essence; when threshold values have to be monitored, small changes in elemental concentrations have to be followed, short transient signals have to be used for quantification or reference materials should be developed. In such cases, often samples are digested to allow for an analysis of the dissolved material with a much better accuracy of $2-5 \%$ while position resolved information is sacrificed.

One important prerequisite or condition for an accurate solid state analysis by LA-ICP-MS is the improvement of data by internal standardization, for which a homogeneously distributed matrix element is required for the normalization. ${ }^{5}$ However, most biological samples do not fulfil this criterion appropriately. To overcome this problem the simultaneous ablation of a sample thin section and a polymeric thin film of a homogeneously distributed element ${ }^{25}$ could be used, where the polymer material serves as the correction standard for the normalization. A disadvantage of this method is the timeconsuming and complex preparation of calibration standards at several different concentration levels. ${ }^{25}$ The preparation of matrix-matched standards is also very time consuming and complex ${ }^{26-30}$ and must be especially adaptable to biological samples. In addition to these problems the homogeneity of matrix-matched standards is often insufficient, which decreases the accuracy. ${ }^{20,21}$ The application of very small laser spots below $10 \mu \mathrm{m}$ for the mapping of biomaterials leads to a gain in 
resolution and information but also puts higher demands on the homogeneity of references in the low micrometer or nanometer dimension.

External calibration with certified reference materials is currently almost impossible to perform for the analysis of biological samples, due to the lack of certified reference materials, which match the sample matrix. Therefore an accurate in-house characterization process for self-prepared and matrix matched reference materials, which uses the same technique for quantitative analysis and provides spatially resolved information about the homogeneity of distribution in the tested reference materials, could lead to more accurate results for the analysis of biological samples.

In general, the best accuracy is achieved by isotope dilution mass spectrometry, since measurements of the amount of substance are traceable to the International System of Units (SI). ${ }^{31}$ In LA-ICP-MS for this a spike solution was added to the ablated aerosol, ${ }^{22-24}$ which is a potential source of error, because the spike and sample in this method are subjected to different processes for the generation of the analytical signal. The sample reaches the plasma torch in the form of particles of different sizes, while the spike is introduced in the form of a homogeneously distributed aerosol. This could lead to incorrect isotope ratios, due to the incomplete atomization and ionization of the sample material.

Becker $e$ t al. ${ }^{22}$ and Pickhardt et al. ${ }^{23}$ used a spike solution for ID and an internal standard for normalization. In a few milligrams of platinum nanoclusters $\mathrm{Ag}, \mathrm{Pb}$ and $\mathrm{Tl}$ were determined using internal standardization with the ${ }^{63} \mathrm{Cu} /{ }^{65} \mathrm{Cu}$ isotope ratio and spike solutions of ${ }^{65} \mathrm{Cu},{ }^{109} \mathrm{Ag},{ }^{204} \mathrm{~Pb}$ and ${ }^{203} \mathrm{Tl}$ enriched isotopes. $^{22}$ In NIST glass SRM 612 and NIST apple leaves SRM 1515, uranium concentrations were measured using Th and Ba as the internal standards, respectively, and NIST U350 as the isotope-enriched spike solution. ${ }^{23}$ In these cases the sample material showed a homogeneous distribution of all analysed elements, so mostly the differences in the sensitivity of signal generation in LA-ICP-MS and ICP-MS of liquids contribute to uncertainties and could be eliminated by correction with the internal standard to a great extent. Most biological samples do not show a homogeneous distribution neither of the main nor of the minor components, which does not allow to pick an element as the internal standard for the analysis.

Douglas et al. analysed iron in tissue samples via double IDMS by online addition of a spike solution. They showed that a main uncertainty source was the mass of the spike with a contribution of $29.6 \%$. This indicates nebulisation of a liquid spike solution $^{24}$ as the critical point in this quantification approach.

The influence of different processes on the generation of the analytical signal may be avoided by applying a solid isotopeenriched standard which could be ablated simultaneously with the sample. This approach has the advantage that isotope dilution could be used at each position of mapping for highly accurate quantification.

In this work we therefore combined the advantages of isotope dilution and normalization to a homogeneously distributed correction standard for a novel calibration strategy for laser ablation mass spectrometry. The use of a solid isotope dilution spike leads to a more similar atomization and ionization of the sample and spike, which eliminates a potential source of error in comparison to Becker ${ }^{22}$ and Pickhardt. ${ }^{23}$ The homogeneously distributed solid spike and correction standard are indirectly supplied to the sample using a modified method from Austin. ${ }^{25}$

This method could be used as an in-house characterization process for self-prepared and matrix matched reference materials as well for the accurate analysis of biological samples.

For single ID and double ID experiments a thin section of the sample and of polymeric thin layers are simultaneously and completely ablated by the laser beam (Fig. 1). The polymeric layers with the homogeneously distributed element(s) or isotope(s) are used for normalization and as the spike for isotope dilution. Thus, the analytical signal of the sample and spike is generated through the same process, which enables a more complete or similar atomization and ionization of the sample and spike. Nevertheless, the possibility of formation of different particle size distributions of the sample and spike and the resulting possible incomplete atomization and ionization should not be neglected and has to be checked, e.g. by comparing the sensitivities normalized to the layer thickness of the different single layers (spike and sample) and the double layer (spike + sample). Thereby equal sensitivities of the individual layers indicate a similar ablation behavior. A relatively large spot size and ablation depth of the laser compared to the layer thickness of the spike and sample can reduce the formation of different particle size distributions due to the decreasing energy transfer of the defocused laser beam at deeper ablation depth $^{32}$ and different aspect ratios ${ }^{33}$ of the laser crater.

The ID-procedure allows the correction of changes in the ablation rate, measuring system, sample matrix and is traceable to the SI-system. A requirement to limit the influence of different ablation rates generated by different sample materials is to guarantee a uniform and known layer thickness and a complete ablation of the sample, which leads to a constant ablated volume. The drift of the laser and measuring system is corrected by normalization to the correction standard. Effects of the sample matrix such as signal suppression or enhancement will influence the correction standard and the ID-spike in the same manner, due to the simultaneous ablation of the sample and spike, and can therefore also be corrected.

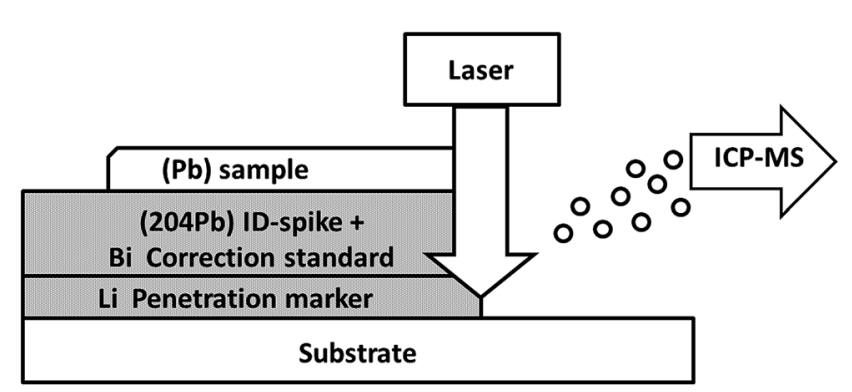

Fig. 1 Schematic illustration of the LA-ICP-ID-MS method for accurate quantification of trace elements in biological tissues (modified from Austin et al. ${ }^{25}$ ). 
Detailed information about isotope dilution can be found in the literature. ${ }^{31,34,35}$

For the calculation of the mass fraction of the analysed element in the sample, modified isotope dilution ${ }^{31}$ formulae were used.

$$
\omega_{x}=\omega_{y, b} \cdot \frac{M_{x} \cdot h_{y}}{M_{b} \cdot h_{x} \cdot a_{x, b}} \cdot \frac{\left(R_{y}-R_{x y}\right)}{\left(R_{x y}-R_{x}\right)}
$$

Eqn (1) (ref. 31) was used for the single isotope dilution experiments. Instead of the mass of the ID-spike and sample, the film thickness $h$ was used. Masses in the numerator and denominator of the equation have been replaced by layer thicknesses because both indicate spike to sample ratios. This exchange is important for the approach discussed here, since thickness measurements could be performed on small samples and reference layers more easily and more accurately as determination of masses. Accurate mass determination is hampered by the tiny amount ablated and variations in the density of the material across the samples, as extensively discussed by Douglas et al. They published another ID-method for the calculation of the actual mass flow rate of the ablated sample by using single IDMS to minimize the challenge in the determination of the accurate ablated mass. ${ }^{24}$ The remaining variables, such as isotope abundancies a, atomic weights $M$, isotope ratios $R$ and mass fraction $\omega$, have not been changed. The index $y$ refers to the ID-spike, $x$ to the sample and $b$ to the reference isotope.

$$
\begin{aligned}
\omega_{x}= & \frac{M_{x} \cdot h_{y}}{a_{x, b} \cdot M_{b} \cdot h_{x}} \cdot \frac{a_{z, b} \cdot M_{b} \cdot h_{z}}{M_{z} \cdot h_{y^{\prime}}} \cdot \omega_{z} \\
& \cdot \frac{\left(R_{y}-R_{x y}\right)}{\left(R_{x y}-R_{x}\right)} \cdot \frac{\left(R_{z y}-R_{z}\right)}{\left(R_{y}-R_{z y}\right)}
\end{aligned}
$$

Eqn (2) (ref. 31) is the modified formula for the double isotope dilution experiments. Again the masses were replaced with the layer thicknesses $h$. In addition, the index $z$ is introduced for the back spike. These formulae are still approximations as differences in the densities of the sample and spike are not considered. Differences in the density have an impact on the actual ablated mass and in these cases the use of the layer thickness will lead to distorted results. If the density of the sample and spike is different, the term $h_{x}, h_{y}$ and $h_{z}$ (layer thicknesses) can be expanded by multiplication of the densities $(\rho)$ of the sample and spike. This introduction of the density will correct for the differences in the actual ablated mass of the sample and spike during the ID-experiment. One possibility for the determination of the density of reference materials and biological samples could be gas pycnometry. ${ }^{36}$

In this paper a novel calibration strategy for the quantitative analysis of trace elements in biological samples and for the characterization of reference materials for the analysis of biological samples by LA-ICP-ID-MS is tested using the single as well as the double ID approach. Due to the lack of certified reference materials for the analysis of biological samples the method development and validation is performed with synthetic polymer materials. A self-prepared synthetic polymeric layer (containing natural lead) and the certified reference material BAM H010 (containing natural lead and mercury) were used. Polymeric samples were chosen because the contained elements are relatively homogeneously distributed within, thus spatially resolved changes of the mass fraction in the samples could be avoided. Since the BAM H010 reference material contains mercury, the isotope ${ }^{204} \mathrm{Hg}$ will generate an isobaric interference for ${ }^{204} \mathrm{~Pb}$, which complicates the analysis and has to be corrected.

\section{Materials and methods}

\section{Preparation of polymeric thin layers}

For the synthesis of the polymeric thin layers a mixture of two UV-curable coatings with two different viscosities was used: 60 parts by weight of the high-viscosity coating no. 54004 and 40 parts by weight of the low-viscosity coating no. 4982700002 (Schekolin AG, Liechtenstein) were mixed. This mixing ratio resulted in a viscosity of $825 \mathrm{mPa}$ s at room temperature and was necessary to produce thin layers with thicknesses of about 4-5 $\mu \mathrm{m}$. BYK-350 (BYK-Gardner GmbH, Geretsried, Germany) was added as an anti-cratering and leveling additive to optimise the mixture's properties. This basis mixture was homogenised with an Ultra-Turrax tube drive control stirring unit (IKA-Werke $\mathrm{GmbH} \&$ Co. KG, Staufen, Germany) for 30 minutes at $4000 \mathrm{rpm}$. After the homogenisation the basis mixture was aliquoted and commercially available $5000 \mathrm{mg} \mathrm{kg}^{-1}$ metal-organic standards of lithium, bismuth, lead (Conostan, Champlain, USA) and isotopic enriched lead were added and homogenised with an Ultra-Turrax tube drive control stirring unit for further 30 minutes at $4000 \mathrm{rpm}$.

For the preparation of the thin layers $(\approx 5 \mu \mathrm{m})$ via knife application, an Automatic Film Applicator S, wire-wound rod 200-0.4 mils (BYK-Gardner GmbH, Geretsried, Germany) and polyester foils (byko-charts, clear polyester 4-mil, $127 \times 194 \mathrm{~mm}$, BYK-Gardner $\mathrm{GmbH}$, Geretsried, Germany) were used. The polyester foils which served as substrates were washed in ethanol before the deposition of the liquid coating mixtures.

After the application of the first polymeric layer, the obtained system was placed in a self-made UV-curing chamber with switched off UV mercury vapour lamps (Philips Actinic BL TL $8 \mathrm{~W} / 10$ 1FM) under an argon gas atmosphere for $30 \mathrm{~min}$, allowing the thin film to flatten the grooves. After the resting time the polymeric layer was cured for 15 min by switching on the UV lamps. All other layers were successively applied onto the already cured layers in an analogous manner, whereby each mixture was repeatedly homogenised immediately before the application.

After the application of each layer, one section of about $1 \mathrm{~cm}^{2}$ was removed for the characterisation of the layer thickness. For the control of the mass fraction of the added elements by liquid analysis, bulk samples $(\approx 500 \mathrm{mg}$ ) of every polymeric thin layer were cured under UV radiation in aluminium trays. For the preparation of the polymeric layer containing natural lead, the commercially available metal-organic standard of lead was used. For the double dilution experiment a microtome thin 
section of the BAM H010 was produced with a thickness of $\approx 13 \mu \mathrm{m}$, because thinner sections were torn apart during the cutting process. The BAM H010 is a acrylonitrile-butadienestyrene-copolymerisate (ABS) bulk material in disc form ( $d=4 \mathrm{~cm}, h=6 \mathrm{~mm}$ ) with certified mass fractions of $\mathrm{Pb}(479 \pm$ $\left.17 \mathrm{mg} \mathrm{kg}^{-1}\right), \mathrm{Br}\left(240 \pm 21 \mathrm{mg} \mathrm{kg}^{-1}\right), \mathrm{Cd}\left(93 \pm 5 \mathrm{mg} \mathrm{kg}^{-1}\right)$ and $\mathrm{Cr}\left(470 \pm 36 \mathrm{mg} \mathrm{kg}^{-1}\right)$. In addition to these analytes a mass fraction of $\approx 415 \mathrm{mg} \mathrm{kg}^{-1}$ of $\mathrm{Hg}$ is also stated as an informative value, due to the possible losses of $\mathrm{Hg}$ by changes in temperature. ${ }^{37}$

\section{Isotope enrichment}

For isotope enrichment in the spike layer, elemental isotope enriched lead with an enrichment of $47.80 \%$ of ${ }^{204} \mathrm{~Pb}$ (EurisoTop GmbH, Saarbrücken, Germany) was used. $100 \mathrm{mg}$ of the metal were digested with subboiled $\mathrm{HNO}_{3}$ and ultrapure water using a high pressure microwave-assisted digestion system (Turbowave, MLS GmbH, Leutkirch, Germany). The digestion solution was evaporated to dryness in an oven at $85{ }^{\circ} \mathrm{C}$. The remaining residue was dissolved in $500 \mu \mathrm{L}$ of ultrapure water and an aliquot of $50 \mu \mathrm{L}$ was added to $5 \mathrm{~mL}$ of the metal-organic lead standard and mixed using the Ultra-Turrax for 30 minutes at $4000 \mathrm{rpm}$. To enable isotope exchange, the mixing was repeated 5 times for a period of 48 hours. This long time period for exchange was set to guarantee equilibrium conditions. The determination of the kinetic parameters for the enrichment of ${ }^{204} \mathrm{~Pb}$ in the spike was not the aim of this study. After the exchange time the isotope enriched metal-organic lead standard was centrifuged to separate the liquid phase from possible solids.

\section{Thin layer characterisation}

The determination of the mass fraction in the thin layers was done via ICP-OES (SPECTRO Arcos, SPECTRO Analytical Instruments $\mathrm{GmbH}$, Kleve, Germany) and external calibration after high pressure microwave-assisted digestion of the polymeric bulk materials with subboiled $\mathrm{HNO}_{3}(\omega=65 \%)$ and subsequent dilution with ultrapure water to $\approx 5 \mu \mathrm{g} \mathrm{kg}{ }^{-1}$.

The homogeneity of the distribution of the added elements ( $\mathrm{Li}, \mathrm{Pb}$ and $\mathrm{Bi}$ ) in the polymeric layers was checked by relative measurements of element distribution after mapping by LA-ICP-MS. Therefore 10 line scans were applied on the thin layers and the relative standard deviation (RSD) values within the lines and between the average values of the lines were calculated after background correction and normalization to ${ }^{13} \mathrm{C}$. The normalization by ${ }^{13} \mathrm{C}$ was used as a control for the homogeneous distribution of all added elements, because carbon as a main matrix element introduced by the polymer should be homogeneously distributed in every layer.

The thickness of every layer was measured by light microscopy (VHX-600, KEYENCE, KEYENCE Deutschland GmbH, Germany) after fixation of every prepared layer (penetration marker-, spike-, blend-, sample-layer) in epoxy resin (EpoThin 2, Buehler, Düsseldorf, Germany). The embedded polymer system (polyester foil with the element/isotope containing thin layers) was cut into three parts with a precision saw (IsoMet 1000
Precision Saw, Buehler, Düsseldorf, Germany) and polished (Phoenix Beta Grinder/Polisher, Buehler, Düsseldorf, Germany). For every layer 75 measurements were carried out on 3 different cross-sections. For the multilayer systems ( $\mathrm{Li}, \mathrm{ID}-\mathrm{Pb}+\mathrm{Bi}$, polymeric layer containing natural lead and $\mathrm{Li}$, ID-Pb $+\mathrm{Bi}$, BAM H010), the layer thicknesses of the individual layers were calculated by subtracting the underlying layers. The images in Fig. 2 show this process for a two-layer system consisting of the layer with the penetration marker $\mathrm{Li}$ and the layer with isotopically enriched lead and $\mathrm{Bi}$ as the internal standard. After preparation of the Li containing layer the thickness was determined by the procedure described above. Afterwards the next layer was synthesized on the top followed by the thickness determination of both layers. Finally the thickness of the ID-spike layer was obtained after subtraction of the layer thickness of the penetration marker from the thickness of the multi-layer of the penetration marker plus the ID-spike.

\section{Experimental setup}

The determination of the isotope ratios and isotope abundancies of lead in the ID-spike layer, the samples (polymeric layer containing natural lead and the reference material BAM H010) and the blend (ID-spike layer + polymeric layer containing natural lead respectively ID-spike layer + BAM H010) was performed by LA-ICP-MS. Therefore 10 line scans were applied on the mentioned layer systems. For mass-bias correction a thallium solution $\left(25 \mu \mathrm{g} \mathrm{kg}^{-1}\right)$ of the reference material NIST SRM 997 was forwarded with a flow rate of $0.17 \mathrm{~mL} \mathrm{~min}^{-1}$ via a Y-piece into the connection tube of the ablated aerosol between the ablation cell and ICP-MS. The exponential law was used for the calculations of true isotope ratios. The mass-bias correction was applied for the single-ID as well as for the double-ID, because in double-ID experiments of solids the matching of isotope ratios of the two different blends is not as simple to achieve as for liquids. The adaptation of the layer thickness of spike, sample and back-spike for matching isotope ratios is more complicated, due to the preparation and characterisation effort for the different layers. Sample-calibrant bracketing for in situ correction was also not performed, because only one sample per time was placed in the laser chamber.

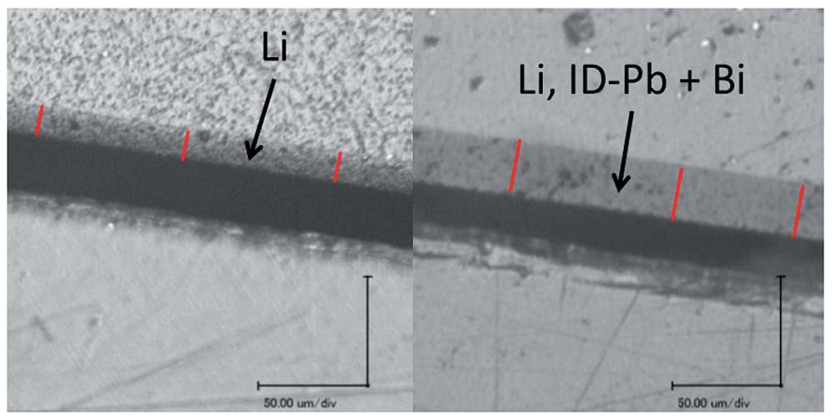

Fig. 2 Determination of the layer thicknesses of the penetration marker $\mathrm{Li}$ (left) and the two-layer system of $\mathrm{Li}$ and ID-Pb + Bi (right). 


\section{LA-ICP-MS}

A nanosecond Nd:YAG deep UV (213 nm) laser ablation system (UP213, New Wave Research, Fremont, USA), coupled to an inductively coupled plasma quadrupole mass spectrometer (XSERIES 2, Thermo Scientific, Waltham, USA) was used to analyse the layer system. For the laser ablation line scans with a spot size of $100 \mu \mathrm{m}$ and $30 \mu \mathrm{m}$, a repetition rate of $20 \mathrm{~Hz}$, a power density of $80 \%\left(\sim 4 \mathrm{~J} \mathrm{~cm}^{-2}\right)$ and a line scan speed of $5 \mu \mathrm{m} \mathrm{s}^{-1}$ were set. The laser beam was focused at the sample surface. The ablated material was transported to the ICP-MS by using helium 5.0 as the carrier gas with a flow rate of $\sim 1.1 \mathrm{~L} \mathrm{~min}^{-1}$.

The analytes $\left({ }^{7} \mathrm{Li},{ }^{13} \mathrm{C},{ }^{203} \mathrm{Tl},{ }^{200} \mathrm{Hg},{ }^{204} \mathrm{~Pb},{ }^{205} \mathrm{Tl},{ }^{206} \mathrm{~Pb},{ }^{207} \mathrm{~Pb}\right.$, ${ }^{208} \mathrm{~Pb}$ and ${ }^{209} \mathrm{Bi}$ ) were monitored during the experiment by ICP-MS (Rf power $1400 \mathrm{~W}$, plasma gas flow $13.0 \mathrm{~L} \mathrm{~min}^{-1}$, auxiliary gas flow $0.8 \mathrm{~L} \mathrm{~min}^{-1}$, nebuliser gas flow $1.0 \mathrm{~L} \mathrm{~min}^{-1}$, dwell time $10 \mathrm{~ms}$; peak hopping mode). Line scans with a length of $3500 \mu \mathrm{m}$ (2000 scans on plateau per isotope) were averaged for the ID-experiment.

\section{Results and discussion}

\section{Characterisation of the thin layers}

For all examined elements, RSD values less than $7 \%$ within a single line have been obtained for the distribution of the elements in the synthetic polymers after elemental mapping with the LA-ICP-MS, background correction and normalization to ${ }^{13} \mathrm{C}$ and less than $3 \%$ between the 10 lines for a laser spot size of $100 \mu \mathrm{m}$. At a spot size of $30 \mu \mathrm{m}$ the RSD values have increased to less than $10 \%$ within the line and less than $3 \%$ between the lines. These results indicate a homogeneous distribution of all added elements and isotopes (including the enriched ${ }^{204} \mathrm{~Pb}$ ) in the polymeric layers. The determination of the via layer thickness normalized sensitivities of the individual layers showed approximately equal values. This indicates a similar ablation behaviour of the samples and spike and should therefore not influence the experiments.

The actual layer thickness has to be known for the isotope dilution method and was determined using light microscopy. The average determined layer thickness was $4.5 \pm 0.6 \mu \mathrm{m}$. The RSD value of the layer thickness was around $12 \%$ pointing out a homogeneous thickness of all layers, although the measurement by light microscopy was hampered by the poor visibility of the solid-solid interface between the different polymer layers and between the polymer layer and substrate as well as the embedding material.

Through the ICP-OES measurements mass fractions of lead were determined in the spike layer $\left(\omega=357 \pm 8 \mathrm{mg} \mathrm{kg}^{-1}\right)$ and the sample layer $\left(\omega=328 \pm 9 \mathrm{mg} \mathrm{kg}^{-1}\right)$. The mass fraction was needed for the calculation by using the ID-formula.

The results of the layer thickness and mass fraction were then used for calculations applied for the ID procedure.

\section{Single isotope dilution}

For testing of the LA-ICP-ID-MS method, single isotope dilution was performed for a polymeric layer containing lead with natural isotope ratios. The sample $(h=4.6 \pm 0.6 \mu \mathrm{m})$ was placed on the top of the polymer system containing the ID-spike and the correction standard layer $(h=4.4 \pm 0.6 \mu \mathrm{m})$, resulting in a sample to spike ratio of nearly 1 . Simultaneous ablation of the complete system with a thickness of $9 \pm 0.6 \mu \mathrm{m}$ was performed. The measured signals of all lead isotopes were background corrected and normalized to ${ }^{209} \mathrm{Bi}$. A continuous ${ }^{7} \mathrm{Li}$ signal over the whole experiment indicated a sufficient ablation depth. The single ID experiments were performed at laser spot sizes of 100 and $30 \mu \mathrm{m}$, for studying the influence of different laser spot sizes on the limit of detection and the expanded uncertainty. The limit of detection was estimated from the threefold signal background and the sensitivity of the measured isotopes. The detection limits of $2 \mathrm{mg} \mathrm{kg}^{-1}$ at $100 \mu \mathrm{m}$ and $6 \mathrm{mg} \mathrm{kg}^{-1}$ at $30 \mu \mathrm{m}$ laser spot size were achieved in the ID-spike layer with a thickness of $4.4 \mu \mathrm{m}$ for the isotope ${ }^{206} \mathrm{~Pb}$.

Mass-bias correction was performed for the lead isotopes. The abundancies determined for the lead isotopes are shown in Table 1 . The results demonstrate a successful enrichment of ${ }^{204} \mathrm{~Pb}$ in the spike layer and indicate a homogeneous/uniform mixing between the ablated ID-spike and sample during the measurement. This is also confirmed by the relatively stable lead isotope raw signals during the whole experiment (compare Fig. 3) and the via ${ }^{209} \mathrm{Bi}$ normalized RSD values of the lead isotopes of less than $7 \%$ within a single line and less than $3 \%$ between the 10 measured lines for a spot size of $100 \mu \mathrm{m}$. For a $30 \mu \mathrm{m}$ spot size, the RSD values slightly increased to less than $10 \%$ within the line and less than $3 \%$ between the lines. $\mathrm{Via}^{209} \mathrm{Bi}$ normalized lead isotope signals were also used for the calculation of the isotope ratios and abundancies for the ID-experiment.

The results of the single isotope dilution of the sample are shown in Table 2. The average mass fraction of the observed isotope ratios leads to accurate values close to the results obtained from the liquid ICP-OES analysis of the sample. The expanded uncertainty $(U)$, with a coverage factor $k=2$, and the confidence interval (CI) were used for calculating inaccuracy. The recovery rate for $\mathrm{Pb}$ was near $100 \%$.

\section{Double isotope dilution}

After the successful single isotope dilution experiment the next step was the testing of a double dilution experiment using the CRM BAM $\mathrm{H} 010$ as the sample with $479 \mu \mathrm{g} \mathrm{g}^{-1} \mathrm{~Pb}$ and $415 \mu \mathrm{g} \mathrm{g}^{-1} \mathrm{Hg}$ (informative value). A microtome thin section $(h=13.2 \pm 0.3 \mu \mathrm{m})$ was placed on the ID-spike layer

Table 1 Average isotope abundancies (\%) in the sample, ID-spike and blend with standard errors $( \pm \mathrm{SE})$

\begin{tabular}{lrrr}
\hline & \multicolumn{3}{l}{ Isotope abundancies } \\
\cline { 2 - 4 } Isotope & \multicolumn{1}{c}{ Sample/\% } & \multicolumn{1}{c}{ ID-spike/\% } & \multicolumn{1}{c}{ Blend/\% } \\
\hline${ }^{204} \mathrm{~Pb}$ & $1.42 \pm 0.01$ & $15.70 \pm 0.05$ & $8.73 \pm 0.04$ \\
${ }^{206} \mathrm{~Pb}$ & $25.02 \pm 0.03$ & $24.49 \pm 0.07$ & $24.84 \pm 0.04$ \\
${ }^{207} \mathrm{~Pb}$ & $21.47 \pm 0.06$ & $18.24 \pm 0.05$ & $19.87 \pm 0.03$ \\
${ }^{208} \mathrm{~Pb}$ & $52.09 \pm 0.07$ & $41.57 \pm 0.06$ & $46.55 \pm 0.07$
\end{tabular}




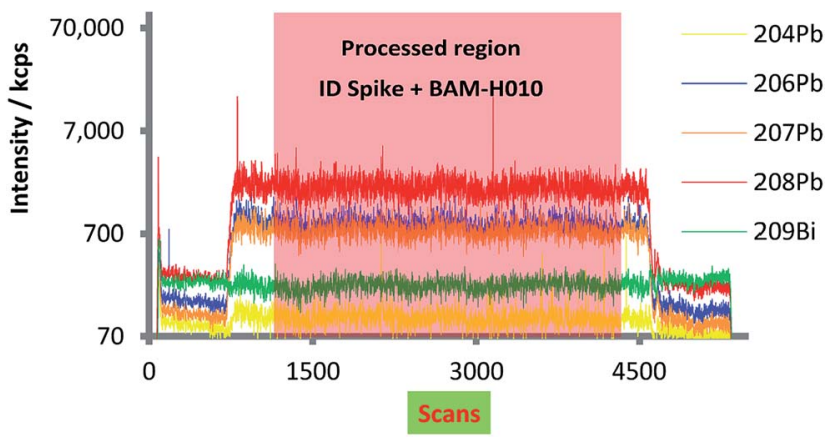

Fig. 3 Logarithmic raw signal profiles of lead and bismuth isotopes during the double spike LA-ICP-ID-MS experiment with CRM BAM H010 as the sample.

Table 2 Comparison of the averaged mass fraction obtained for lead by LA-ICP-ID-MS with expanded uncertainty $( \pm U)$ and by ICP-OES with confidence interval $( \pm \mathrm{Cl})$

\begin{tabular}{ll}
\hline LA-ICP-ID-MS & $\begin{array}{l}\text { Mass fraction } \\
\omega(\mathrm{Pb}) \text { per } \mathrm{mg} \mathrm{kg}\end{array}$ \\
\hline${ }^{204} \mathrm{~Pb} /{ }^{208} \mathrm{~Pb}$ & $332 \pm 33$ \\
${ }^{204} \mathrm{~Pb} /{ }^{207} \mathrm{~Pb}$ & $330 \pm 33$ \\
${ }^{204} \mathrm{~Pb} /{ }^{206} \mathrm{~Pb}$ & $325 \pm 33$ \\
$\bar{X}$ & $329 \pm 33$ \\
ICP-OES & $328 \pm 9$
\end{tabular}

( $h=4.4 \pm 0.6 \mu \mathrm{m}$ ) and the polymeric layer containing natural lead ( $h=4.6 \pm 0.6 \mu \mathrm{m}$ ) was used as the back-spike to characterise the ID-spike. In this experiment the sample to spike ratio was around 4. The laser ablation was performed as mentioned above; in addition, mercury correction for the ${ }^{204} \mathrm{~Pb}$ isotope was applied by measuring the ${ }^{200} \mathrm{Hg}$ isotope. The double ID experiment was performed at a spot size of $100 \mu \mathrm{m}$.

Fig. 3 illustrates the raw signal profile of ${ }^{204} \mathrm{~Pb},{ }^{206} \mathrm{~Pb}$, ${ }^{207} \mathrm{~Pb},{ }^{208} \mathrm{~Pb}$ and ${ }^{209} \mathrm{Bi}$ during the measurement of a single line scan on the multi-layer system containing the ID-spike underneath the BAM H010 microtome thin section. All observed signals remained constant during the line scan, indicating a uniform material ablation and a homogeneous mixing of the ablated blend. The results of the double isotope dilution are listed in Table 3. Again the average mass

Table 3 Comparison of averaged mass fractions $( \pm U$ ) obtained by LAICP-ID-MS and certified mass fraction for lead in the reference material BAM H010

\begin{tabular}{ll}
\hline LA-ICP-ID-MS & $\begin{array}{l}\text { Mass fraction } \\
\omega(\mathrm{Pb}) \text { per } \mathrm{mg} \mathrm{k}^{-1}\end{array}$ \\
\hline${ }^{204} \mathrm{~Pb} /{ }^{208} \mathrm{~Pb}$ & $483 \pm 38$ \\
${ }^{204} \mathrm{~Pb} /{ }^{207} \mathrm{~Pb}$ & $480 \pm 38$ \\
${ }^{204} \mathrm{~Pb} /{ }^{206} \mathrm{~Pb}$ & $484 \pm 38$ \\
$\bar{X}$ & $483 \pm 38$ \\
Certified value BAM H010 & $479 \pm 17$
\end{tabular}

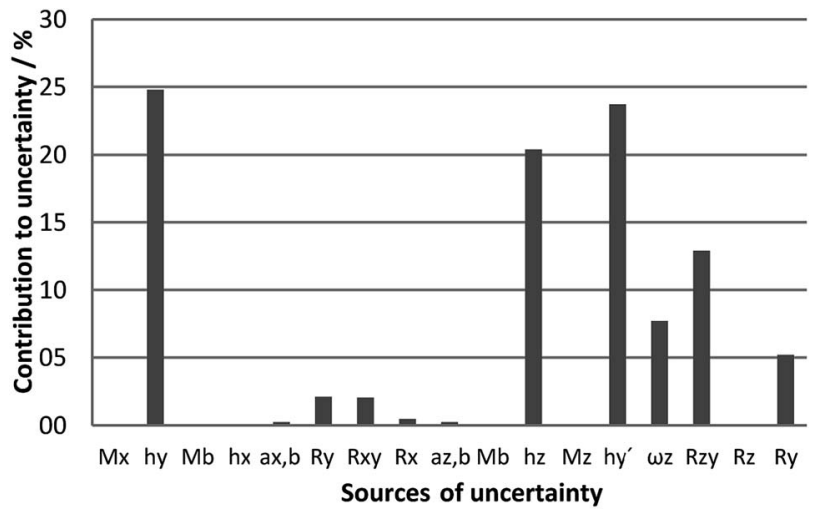

Fig. 4 Sources of uncertainty and their contribution to expanded uncertainty. Uncertainty of layer thickness is responsible for over $75 \%$ of the total budget.

fraction of the three observed isotope ratios leads to accurate values close to the certified values of the BAM reference material. The recovery rate showed a value close to $100 \%$.

\section{Uncertainty calculation}

The uncertainty calculation was carried out according to the very conservative approach by Kragten ${ }^{38}$ using the single and double dilution formula. As input variables the standard errors (SEs) and the confidence interval (CI) for the mass fraction of the ID-spike or back-spike were used. The indicated uncertainty is the expanded uncertainty $(U)$ with a coverage factor $k=2$.

For the single isotope dilution, the expanded uncertainty was around $11 \%$ and for the double isotope dilution a lower value at around $8 \%$ was obtained. The analysis of the fraction of the individual parameters in the uncertainty budget (Fig. 4) showed that the main sources of the uncertainty were the layer thicknesses of the ID-spike, the sample layer and the back-spike layer with $25 \%$ each. Measurements of layer thicknesses of a few micrometres are difficult to be performed by light microscopy. Eventually profilometry could be used as an alternative in the future or colouring of the polymer matrix could help measure each layer individually with higher accuracy instead of using subtraction of different thicknesses as done in this work. With this, the uncertainty of the methods could probably be reduced in the future below $5 \%$, thus getting close to values achieved by liquid analysis so far. The relatively large contribution of $R_{z y}$ and $R_{y}$ to the uncertainty is caused by poor statistics. The back-spike blend and the corresponding spike were measured only 3 times instead of 10 times, resulting in a value twice that of the standard error and a three times larger contribution to the uncertainty.

Investigations show that the influence of varying laser spot sizes does not affect the expanded uncertainty, when the measured signals are significantly higher than the detection limit. 


\section{Conclusions}

The characterisation of the prepared thin reference layers indicated a homogeneous distribution of all added elements, including the isotopically enriched lead as well as constant thicknesses of all layer materials synthesized. The tested isotope dilution method for LA-ICP-MS has shown its potential for the accurate quantification of averaged mass fractions in two test samples (CRM BAM H010 and a polymeric layer containing natural lead). The averaged calculated mass fractions of lead in both test samples showed a deviation from the certified value of less than $1 \%$. Single and double isotope dilutions were performed. The expanded uncertainty for single and double isotope dilutions was $11 \%$ and $8 \%$, respectively, using a conservative approach for calculation and a $100 \mu \mathrm{m}$ laser spot size. The two different sample to spike ratios of 1 and 4 tested in this work had only a minor impact on the expanded uncertainty with $11 \%$ instead of $13 \%$ for single ID experiments. The RSD values within single ablation lines of the two different analysed polymeric matrices showed nearly the same values; this indicates an equal signal noise for the different matrices. Smaller laser spot sizes showed a minor effect on the expanded uncertainty as long as the signals were significantly higher than the detection limits of the measured isotopes. Detection limits of $2 \mathrm{mg} \mathrm{kg}{ }^{-1}$ at a laser spot size of $100 \mu \mathrm{m}$ and $6 \mathrm{mg} \mathrm{kg}^{-1}$ at $30 \mu \mathrm{m}$ have been determined.

Uncertainties of layer thicknesses were identified to be the main contributors to the overall uncertainty budget, thus improving the quality of layer thickness determination by colouring the polymers or using profilometry will reduce expanded uncertainty significantly in the future. By using time-of-flight or multi-collector mass spectrometers and femtosecond laser systems together, the expanded uncertainty could be reduced to less than $5 \%$. This method may be used in the future for highly accurate spatially resolved quantification via LA-ICP-ID-MS.

The total time consumption for the preparation and characterization of the ID-spike layers takes around one week. A large part of this time is consumed by the initial preparation of the polymer mixtures (which could be saved for further experiments) and the characterization of the reference layers. The production of one spike layer with an area of $100 \mathrm{~cm}^{2}$ takes only about 2 hours and can be repeated frequently due to the good storability of the polymer mixtures. The analysis time of different samples using the method proposed here requires about twice the time compared with typical laser ablation experiments due to the lower scan speed, which is necessary to guarantee a complete ablation of the sample and ID-spike. Thus this calibration strategy is comparable to the preparation and characterization of matrix matched standards by other approaches and may represent a powerful alternative for accurate quantification via LA-ICP-MS.

\section{Acknowledgements}

The authors gratefully acknowledge support from Anja Dreyer in the preparation of the polymeric thin layers and René Frankfurter, Fabian Zimmermann, Anica Weller and Nicola
Boyer for assistance with the experiments and helpful discussions.

\section{Notes and references}

1 J. S. Becker, R. C. Dietrich, A. Matusch, D. Pozebon and V. Dressler, Spectrochim. Acta, Part B, 2008, 67, 1248-1252.

2 A. Bogaerts, Z. Y. Chen, R. Gijbels and A. Vertes, Spectrochim. Acta, Part B, 2003, 58, 1867-1893.

3 M. Siebold, P. Leidich, M. Bertini, G. Deflorio, J. Feldmann, E. M. Krupp, E. Halmschlager and S. Woodward, Anal. Bioanal. Chem., 2012, 402, 3323-3331.

4 S. Pattberg and R. Matschat, J. Anal. Chem., 1999, 364, 410416.

5 T. Leveque, Y. Capowiez, E. Schreck, C. Mazzia, M. Auffan, Y. Foucault, A. Austruy and C. Dumat, Environ. Pollut., 2013, 179, 232-241.

6 R. Delfino, M. Altissimo, R. H. Menk, R. Alberti, T. Klatka, T. Frizzi, A. Longoni, M. Salomè, G. Tromba, F. Arfelli, M. Clai, L. Vaccari, V. Lorusso, C. Tiribelli and L. Pascolo, Clin. Exp. Pharmacol. Physiol., 2011, 38, 834-845.

7 R. G. Figueroa, E. Lozano and G. Bongiovanni, Rev. Mex. Fis., 2013, 59, 292-295.

8 M. West, A. T. Ellis, P. J. Potts, C. Streli, C. Vanhoof and P. Wobrauschek, J. Anal. At. Spectrom., 2014, 29, 1516.

9 O. Hachmöller, A. G. Buzanich, M. Aichler, M. Radtke, D. Dietrich, K. Schwamborn, L. Lutz, M. Werner, M. Sperling, A. Walch and U. Karst, Metallomics, 2016, DOI: 10.1039/c6mt00001k.

10 D. J. Hare, E. J. New, M. D. de Jongee and G. McColl, Chem. Soc. Rev., 2015, 44, 5941-5958.

11 K. L. Moore, E. Lombi, F.-J. Zhao and C. R. M. Grovenor, Anal. Bioanal. Chem., 2012, 402, 3263-3273.

12 T. Eybe, J. N. Audinot, T. Bohn, C. Guignard, H. N. Migeon and L. Hoffmann, J. Appl. Microbiol., 2008, 105, 1502-1510.

13 M. K. Passarelli and N. Winograd, Biochim. Biophys. Acta, 2011, 1811, 976-990.

14 J. C. Vickerman and N. Winograd, Int. J. Mass Spectrom., 2015, 377, 568-579.

15 J. S. Becker, Int. J. Mass Spectrom., 2010, 289, 65-75.

16 J. S. Becker, A. Matusch and B. Wu, Anal. Chim. Acta, 2014, 835, 1-18.

17 D. A. Frick, C. Giesen, T. Hemmerle, B. Bodenmiller and D. Günther, J. Anal. At. Spectrom., 2015, 30, 254-259.

18 B. Klug, A. Specht and W. J. Horst, J. Exp. Bot., 2011, 62, 5453-5462.

19 B. Wua, M. Zoriy, Y. Chena and J. S. Becker, Talanta, 2009, 78, 132-137.

20 B. Wua, Y. Chena and J. S. Becker, Anal. Chim. Acta, 2009, 633, 165-172.

21 J. S. Becker, Int. J. Mass Spectrom., 2010, 289, 65-75.

22 J. S. Becker, C. Pickhardt and W. Pompe, Int. J. Mass Spectrom., 2004, 237, 13-17.

23 C. Pickhardt, A. V. Izmer, M. V. Zoriy, D. Schaumlöffel and J. S. Becker, Int. J. Mass Spectrom., 2006, 248, 136-141.

24 D. N. Douglas, J. O'Reilly, C. O'Connor, B. L. Sharp and H. Goenaga-Infante, J. Anal. At. Spectrom., 2016, 31, 270-279. 
25 C. Austin, D. Hare, T. Rawling, A. M. McDonagh and P. Doble, J. Anal. At. Spectrom., 2010, 25, 722-725.

26 J. Dobrowolska, M. Dehnhardt, A. Matusch, M. Zoriy, N. Palomero-Gallagher, P. Koscielniak, K. Zilles and J. S. Becker, Talanta, 2008, 74, 717-723.

27 J. S. Becker, M. V. Zoriy, C. Pickhardt, N. Palomero-Gallagher and K. Zilles, Anal. Chem., 2005, 77, 3208-3216.

28 M. Zoriy, A. Matusch, T. Spruss and J. S. Becker, Int. J. Mass Spectrom., 2007, 260, 102-106.

29 J. S. Becker, M. V. Zoriy, M. Dehnhardt, C. Pickhardt and K. Zilles, J. Anal. At. Spectrom., 2005, 20, 912-917.

30 J. S. Becker, A. Matusch, C. Depboylu, J. Dobrowolska and M. V. Zoriy, Anal. Chem., 2007, 79, 6074-6080.
31 J. Vogel and W. Pritzkow, MAPAN, 2010, 25, 135-164.

32 R. E. Russo, X. Mao, H. Liu, J. Gonzalez and S. S. Mao, Talanta, 2002, 57, 425-451.

33 H. C. Liu, O. V. Borisov, X. L. Mao, R. E. Russo and S. Shuttleworth, Appl. Spectrosc., 2000, 54, 1435-1442.

34 K. G. Heumann, Int. J. Mass Spectrom., 1992, 118, 575-592. 35 J. D. Fassett and P. J. Paulsen, Anal. Chem., 1989, 61, 643649.

36 U. Stange, M. Scherf-Clavel and H. Gieseler, J. Pharm. Sci., 2013, 102, 4087-4099.

37 BAM Federal Institute for Materials Research and Testing, Certified Reference Material BAM-H010, 2013.

38 J. Kragten, Analyst, 1994, 119, 2161-2166. 